\title{
Improvement of PEP-II Linear Optics with a MIA-Derived Virtual Accelerator
}

\author{
Ben Cerio \\ Office of Science, Science Undergraduate Laboratory Internship (SULI) \\ Colgate University \\ Stanford Linear Accelerator Center \\ Stanford, California
}

August 18, 2006

Prepared in partial fulfillment of the requirements of the Office of Science, Department of Energy's Science Undergraduate Laboratory Internship under the direction of Yiton Yan in the Beam Physics Department at the Stanford Linear Accelerator Center.

\section{Participant:}

Research Advisor: 


\section{Table of Contents}

Abstract........................................................... iii.

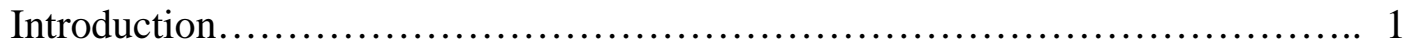

Materials and Methods.................................................... 2

Results............................................................ 7

Discussion and Conclusion............................................... 9

Acknowledgements................................................. 10

References........................................................ 10

Figures........................................................... 11 


\begin{abstract}
Improvement of PEP-II Linear Optics with a MIA-Derived Virtual Accelerator. BEN CERIO (Colgate University, Hamilton, NY 13346, USA) YITON YAN (Stanford Linear Accelerator Center, Stanford, CA 94309, USA).
\end{abstract}

In several past studies, model independent analysis, in conjunction with a virtual accelerator model, has been successful in improving PEP-II linear geometric optics. In many cases, optics improvement yielded an increase in machine luminosity. In this study, an updated characterization of linear optics is presented. With the PEP-II beam position monitor (BPM) system, four independent beam centroid orbits were extracted and used to determine phase advances and linear Green’s functions among BPM locations. A magnetic lattice model was then constructed with a singular value decompositionenhanced least-square fitting of phase advances and Green’s functions, which are functions of quadrupole strengths, sextupole feed-downs, as well as BPM errors, to the corresponding measured quantities. The fitting process yielded a machine model that matched the measured linear optics of the real machine and was therefore deemed the virtual accelerator. High beta beat, as well as linear coupling, was observed in both LER and HER of the virtual accelerator. Since there was higher beta beating in LER, focus was shifted to the improvement of this ring. By adjusting select quadrupoles of the virtual LER and fitting the resulting beta functions and phase advances to those of the desired lattice, the average beta beat of the virtual machine was effectively reduced. The new magnet configuration was dialed into LER on August 10, 2006, and beta beat was reduced by a factor of three. After fine tuning HER to match the improved LER for optimal collision, a record peak luminosity of $12.069 \times 10^{33} \mathrm{~cm}^{-2} \mathrm{~s}^{-1}$ was attained on August 16, 2006. 


\section{INTRODUCTION}

PEP-II is an electron-positron collider with asymmetric energy storage rings: the high energy ring (HER) which stores electrons at $9.0 \mathrm{GeV}$, and the low energy ring (LER) which stores positrons at $3.1 \mathrm{GeV}$. These two rings intersect at the interaction point (IP), where the BaBar detector is located. PEP-II, a complex and dynamic machine, must be regularly upgraded to remain at the frontier of particle physics research.

The improvement of linear optics in PEP-II has, in the past, resulted in greater luminosity. After taking BPM measurements, it is possible to extract 4 independent beam centroid orbits. These 4 orbits are then inputted into a model independent analysis MIA for extracting critical linear optics parameters [1,2]. With the ideal lattice as the initial model, the parameters of the model are fitted with a singular value decomposition (SVD)enhanced least square fitting technique to the corresponding measurement-derived quantities, yielding a computer virtual machine that matches the linear optics of PEP-II LER or HER. The strengths of select magnetic quadrupoles of the virtual machine are varied and a new magnet configuration that minimizes beta beating and linear coupling is determined. The improved magnet configuration may then be applied to PEP-II, thus improving the linear optics and perhaps the luminosity.

For the past several months, PEP-II Operations was pushing current as a strategy

to increase luminosity. Although this strategy was successful in breaking peak luminosity records, we saw an increase in beta beat in LER and HER. This degradation of linear optics called for an updated MIA-derived virtual accelerator from which we could determine a beta-beat-mitigating solution. 
In this study, we continued to use MIA as a tool for optimization of PEP-II optics. We discuss the process of constructing a virtual machine derived from BPM measurements. We then present a comparison of the virtual and ideal lattice optics, with a focus on betatron amplitude and phase, linear coupling, and IP optics characterization. We conclude with a discussion of the creation of an improved virtual LER that minimizes beta beat and the subsequent improvement of the real machine.

\section{MATERIALS AND METHODS}

\section{BPM DATA ACQUISITION}

In PEP-II HER there are 38 single view horizontal (x), 40 single view vertical (y), and 215 double view BPMs, corresponding to a maximum of $253 \mathrm{x}$ data and $255 \mathrm{y}$ data. The LER has 46 single view horizontal, 44 single view vertical, and 229 double view BPMs. PEP-II BPMs are capable of acquiring turn-by-turn data at a resolution of a few microns, allowing for precise orbit measurements. With a single bunch in HER (LER), we resonantly excited the beam with the transverse feedback kicker in straight 4 at the horizontal betatron tune for 1024 turns, resulting in a 1024-by-253 (275) matrix of x data and a 1024-by-255 (273) matrix of y data. We also excited the beam vertically at the vertical betatron tune and stored another set of $\mathrm{x}$ and y BPM buffer data.

\section{EXTRACTION OF ORBITS}

We performed a tune-matched fast Fourier transform on the columns of the raw BPM buffer data, which allowed us to extract the resonant betatron mode. The resonant mode contains two degrees of freedom, an imaginary part and a real part, which represent the sine-like orbit and cosine-like orbit, respectively. Since this was done for transverse 
directions ( $\mathrm{x}$ and $\mathrm{y}$ ) and for both beam excitations (horizontal and vertical), we obtained a four orbit matrix Z at each BPM location. For an arbitrary location a,

$$
Z^{a}=\left[\begin{array}{llll}
\vec{Z}_{1}^{a} & \vec{z}_{2}^{a} & \vec{Z}_{3}^{a} & \vec{Z}_{4}^{a}
\end{array}\right]=\left[\begin{array}{cccc}
x_{1}^{a} & x_{2}^{a} & x_{3}^{a} & x_{4}^{a} \\
x_{1}^{\prime a} & x_{2}^{\prime a} & x_{3}^{\prime a} & x_{4}^{\prime a} \\
y_{1}^{a} & y_{2}^{a} & y_{3}^{a} & y_{4}^{a} \\
y_{1}^{\prime a} & y_{2}^{\prime a} & y_{3}^{\prime a} & y_{4}^{\prime a}
\end{array}\right]
$$

where $\vec{z}_{1}^{a}$ and $\vec{z}_{2}^{a}$ are orbits from horizontal excitation, $\vec{z}_{3}^{a}$ and $\vec{z}_{4}^{a}$ are orbits from vertical excitation, and $x^{\prime}$ and $y^{\prime}$ have not been measured. Figure 1 shows four independent beam orbits. Note the coupling between the $\mathrm{x}$ and y orbits near IP (BPM 155-156). PHASE ADVANCES AND LINEAR GREEN'S FUNCTIONS

The independent orbits derived from measurement allowed us to calculate betatron phase advances and linear Green's functions from which we determined critical linear optics parameters. To calculate the phase $\mu$ at an arbitrary location a, we have

$$
\mu_{a}=\tan ^{-1}\left[\frac{\operatorname{Im}\left(x^{a}\right)}{\operatorname{Re}\left(x^{a}\right)}\right],
$$

where $x_{a}$ is the Fourier-transformed orbit at the betatron resonance. Phase advances at adjacent locations were simply calculated by subtraction [3]. This calculation assumed no BPM errors. BPM gains were canceled in the ratio; however, cross coupling remained and phase advances were therefore corrected after fitting of the virtual model for which BPM cross coupling was a fitting variable.

MIA also determined linear Green’s functions to which we fit the corresponding model Green’s functions. For arbitrary lattice locations a and b, we defined a linear map $Z^{b}=R^{a b} Z^{a}$, where $R^{a b}$ is the transfer matrix from a to b, and $Z^{a}$ and $Z^{b}$ are the 
orbit matrices at these locations. Since transfer matrices are canonical transformations, we applied the symplectic condition, $R^{a b T} S R^{a b}=S$, where

$$
S=\left[\begin{array}{cc}
S_{2 \times 2} & 0 \\
0 & S_{2 \times 2}
\end{array}\right] \text { with } S_{2 \times 2}=\left[\begin{array}{cc}
0 & 1 \\
-1 & 0
\end{array}\right] \text {. }
$$

Using this condition, we defined an invariant matrix Q, where

$$
Q=Z^{b T} S Z^{b}=\left(R^{a b} Z^{a}\right)^{T} S R^{a b} Z^{a}=Z^{a T} S Z^{a} .
$$

The Green’s functions are merely transfer matrix elements and can be expressed as follows [5]:

$$
\begin{aligned}
& \left(x_{1}^{a} x_{2}^{b}-x_{2}^{a} x_{1}^{b}\right) / Q_{12}+\left(x_{3}^{a} x_{4}^{b}-x_{4}^{a} x_{3}^{b}\right) / Q_{34}=R_{12}^{a b}, \\
& \left(x_{1}^{a} y_{2}^{b}-x_{2}^{a} y_{1}^{b}\right) / Q_{12}+\left(x_{3}^{a} y_{4}^{b}-x_{4}^{a} y_{3}^{b}\right) / Q_{34}=R_{32}^{a b}, \\
& \left(y_{1}^{a} x_{2}^{b}-y_{2}^{a} x_{1}^{b}\right) / Q_{12}+\left(y_{3}^{a} x_{4}^{b}-y_{4}^{a} x_{3}^{b}\right) / Q_{34}=R_{14}^{a b}, \\
& \left(y_{1}^{a} y_{2}^{b}-y_{2}^{a} y_{1}^{b}\right) / Q_{12}+\left(y_{3}^{a} y_{4}^{b}-y_{4}^{a} y_{3}^{b}\right) / Q_{34}=R_{34}^{a b} .
\end{aligned}
$$

The above expressions, however, assume that the BPM measurements are perfect. In fact, when we fitted our model to the measurement-derived Green's functions, we had to account for BPM gains and cross-plane coupling:

$$
\begin{aligned}
& R_{12}^{a b}=g_{x}^{b} \Re_{12}^{a b} g_{x}^{a}+g_{x}^{b} \Re_{14}^{a b} \theta_{x y}^{a}+\theta_{x y}^{b} \Re_{32}^{a b} g_{x}^{a}+\theta_{x y}^{b} \Re_{34}^{a b} \theta_{x y}^{a}, \\
& R_{32}^{a b}=g_{y}^{b} \Re_{32}^{a b} g_{x}^{a}+g_{y}^{b} \Re_{34}^{a b} \theta_{x y}^{a}+\theta_{y x}^{b} \Re_{12}^{a b} g_{x}^{a}+\theta_{y x}^{b} \Re_{14}^{a b} \theta_{x y}^{a}, \\
& R_{14}^{a b}=g_{x}^{b} \Re_{14}^{a b} g_{y}^{a}+g_{x}^{b} \Re_{12}^{a b} \theta_{y x}^{a}+\theta_{x y}^{b} \Re_{34}^{a b} g_{y}^{a}+\theta_{x y}^{b} \Re_{32}^{a b} \theta_{y x}^{a}, \\
& R_{34}^{a b}=g_{y}^{b} \Re_{34}^{a b} g_{y}^{a}+g_{y}^{b} \mathfrak{R}_{32}^{a b} \theta_{y x}^{a}+\theta_{y x}^{b} \Re_{14}^{a b} g_{y}^{a}+\theta_{y x}^{b} \Re_{12}^{a b} \theta_{y x}^{a},
\end{aligned}
$$

where $g_{x}$ and $g_{y}$ are BPM gains, and $\theta_{x y}$ and $\theta_{y x}$ are BPM cross coupling multipliers [5].

$R$ ' $s$ are the linear Green's functions in the measurement frame, while $\mathfrak{R}$ 's are the corresponding quantities of the model that are corrected accordingly. 


\section{SVD-ENHANCED LEAST-SQUARE FITTING}

After determining phase advances and linear Green's functions with MIA, we were in a position to construct a machine model by fitting model parameters to these phase advances and linear Green’s functions derived from measurement. We organized quantities into vectors and defined the fundamental fitting relationship $\vec{Y}(\vec{X})=\vec{Y}_{m}$, where the elements of $\vec{Y}_{m}$ are the Green's functions and phase advances derived from measurement and the elements of $\vec{Y}$ are the corresponding model quantities which depend on $\vec{X}$ (the BPM gains and cross couplings, the quadrupole strengths, and the sextupole feed-downs). We began the fitting process with reasonable fitting variables, denoted $\vec{x}_{0}$, which were derived from the design (ideal) lattice. Letting $\vec{X}=\vec{x}_{0}+\vec{x}$, we Taylor expanded $\vec{Y}(\vec{X})$, obtaining

$$
\vec{Y}\left(\vec{x}_{0}+\vec{x}\right)=\vec{Y}\left(\vec{x}_{0}\right)+M \vec{x}+\vec{\eta}(\vec{x})=\vec{Y}_{m}
$$

where $M=\frac{d \vec{Y}}{d \vec{x}}\left(\vec{x}_{0}\right)$, and $\vec{\eta}(\vec{x})$ represents the nonlinear terms which grow increasingly negligible with each iteration and can, therefore, be ignored, giving

$$
M \vec{x}=\vec{Y}_{m}-\vec{Y}\left(\vec{x}_{0}\right) \equiv \vec{b}
$$

The least squares solution, $\vec{x}=\left(M^{T} M\right)^{-1} M^{T} \vec{b}$, diverged due to degeneracies, calling for an SVD-enhanced Least-Square fitting process that identified dominant SVD modes [6]. When $\vec{b}$, the residuals, were sufficiently small, the iteration process stopped, and we obtained a model lattice that matched the lattice of the real accelerator. From this lattice, we calculated the one turn transfer matrices from which we extracted critical optical parameters at each BPM location. 


\section{MANIPULATION OF VIRTUAL MACHINE}

Due to high beta beat and linear coupling in LER, we decided to focus on the optics improvement of this machine. Furthermore, the LER model more closely matched the real machine than HER, implying that the LER model would accurately respond to quadrupole adjustment. We again used SVD-enhanced fitting; however, in this case, we, in a sense, fitted in the reverse direction with the fitting relationship $\vec{Y}_{v m}(\vec{Z})=\vec{Y}_{0}$, where $\vec{Y}_{0}$ represents the optical parameters of the desired machine and $\vec{Y}_{v m}$ represents the corresponding quantities of the virtual machine. The response quantities depended on $\vec{Z}$, which represented the strengths of 36 quadrupoles, including linear trombone quads and local and global skews. We defined the fitting residuals as $\vec{Y}_{v m}-\vec{Y}_{0}$. When these residuals became sufficiently low, the iteration process stopped and we obtained a new virtual accelerator that more closely matched the desired lattice. Before fitting started, we were able to weight optical parameters that we considered to be highly critical in our solution. The program, then, placed an increased emphasis on the reduction of the residuals of these parameters and, as a result, other residuals increased. The challenge was to find a combination of parameter weights that decreased important residuals and left the others unchanged. We determined this combination and obtained an improved virtual accelerator with the magnet configuration at the time that BPM data was taken plus the new magnet strengths. We then created a knob, which characterized this new magnet configuration, in the machine control system. The machine operators dialed this knob into the machine and we took more BPM measurements with which we repeated MIA and determined an updated optics characterization. We compared the linear optics parameters 
of the improved real machine to those of the pre-solution machine to gauge the success of our study.

\section{RESULTS}

\section{LINEAR OPTICS OF VIRTUAL ACCELERATOR}

The following LER results were obtained from MIA measurements taken on August 9, 2006. Although HER MIA data were taken on this date, we decided not to focus on this machine, because the HER is currently under planning for a replacement of its $60^{\circ}$ lattice with a $90^{\circ}$ lattice. Thus, our primary focus was shifted to LER. We removed 5 horizontal view and 6 vertical view BPM data from our LER analysis, and the resulting fitting residuals were sufficiently low. Figure 2 compares linear Green’s functions of the model before fitting with those derived from measurement for LER. The figure clearly displays deviations between the two; these deviations were corrected by our fitting process, and the Green’s functions of the resulting model are shown in figure 3. This figure shows an almost perfect match between the measurement-derived Green’s functions and the model Green's functions.

The betatron phase advances are also model response quantities that are useful in determining goodness-of-fit. Figure 4 displays phase advances derived from measurement and phase advances of the starting model (ideal lattice) for LER. Figure 5 shows betatron phase advances after SVD-enhanced fitting. Again, the figures illustrate that we have obtained a model which, according to our BPM measurements, closely resembles the real machine.

After we obtained sufficiently low Green's function and phase advance fitting residuals, we calculated linear optical parameters from one turn maps. Figure 6 compares 
the beta functions of the ideal lattice and our virtual machine. This plot indicates high beta beating in eigenplane 1 . This figure also shows beta functions in the vicinity of IP for the virtual machine and the ideal machine. In virtual LER, $\beta_{x}^{*}=19.6 \mathrm{~cm}$ and $\beta_{y}^{*}=10.1 \mathrm{~mm}$. While the virtual machine $\beta_{y}^{*}$ is reasonably close to that of the ideal lattice, $\beta_{x}^{*}$ is lower due to beta beat. Displayed in figure 7 is a comparison of the coupling parameters between the virtual machine and the ideal lattice. The figure indicates that linear coupling in the model is greater than coupling in the ideal lattice. Strong coupling, in concert with beta beat, increases beam size and consequently degrades luminosity. Therefore, it was our goal to reduce these optical parameters in our model and then apply the new magnet configuration to the real machine.

\section{LINEAR OPTICS OF IMPROVED VIRTUAL ACCELERATOR}

Since the virtual accelerator indicated high beta beat, we placed an appropriately high weight on beta functions in both $\mathrm{x}$ and $\mathrm{y}$ directions during SVD-enhanced fitting of the virtual accelerator to the ideal lattice. Increased weight on beta functions successfully reduced the residuals of this parameter, but increased the residuals of other parameters. We altered the parameter weights and fitted several times before reaching an acceptable solution. In our solution, beta beat was reduced by a factor of $\sim 3$, and other parameters remained within a reasonable range. Figure 8 displays plots of beta functions for the improved virtual accelerator. Note the reduction of beta beat in $\mathrm{x}$ and the increase in the horizontal beta function at IP.

Figure 9 compares linear coupling of the improved machine and the ideal lattice. Since we did not place a high weight on coupling parameters in the fitting process, we 
did not expect a significant change in coupling. Comparing figures 7 and 9, it is apparent that coupling ellipse tilt ratios were in fact reduced in the improved virtual accelerator.

After dialing the improved virtual accelerator into the machine, we repeated model independent analysis and constructed a new virtual accelerator in order to check that our new magnet configuration was indeed successful in improving linear optics. To avoid confusion we will call this virtual accelerator the post-solution virtual machine. Figure 10 displays the beta functions of the post-solution virtual machine. Note the decrease in beta beat, which we predicted with the improved virtual accelerator. The post-solution virtual accelerator indicated an increase in $\beta_{x}^{*}$ that was greater than predicted, and a $10 \%$ increase in $\beta_{y}^{*}$, which agreed with predictions.

\section{DISCUSSION AND CONCLUSION}

In this study, after a systematic removal of noisy BPM data, we successfully constructed a virtual accelerator that matched the linear optics of PEP-II LER. By manipulating the virtual lattice and placing appropriate emphasis on problematic linear optical parameters, we found a magnet configuration that improved the beta beat of the virtual LER. When applied to the real machine, the beta beat decreased by a factor of $~ 3$ as predicted (see figure 10). The new solution yielded an increase in beta functions at IP that was within the range of prediction (compare figures 8 and 10) given that the presolution orbit was not restored after the new magnet configuration was dialed in to the machine. The HER lattice was fine-tuned after the dial in of the new LER solution, and we observed improvement in the performance of the machine. During machine

development on August 16, 2006, PEP-II reached a record peak luminosity of 
$12.069 \times 10^{33} \mathrm{~cm}^{-2} \mathrm{~s}^{-1}$. Furthermore, following the dial in of the new solution, the average luminosity integration increased, and the machine reached the current run goal of $100 \mathrm{fb}^{-1}$ before the anticipated date.

\section{ACKNOWLEDGEMENTS}

I would like to extend my thanks to my advisor, Yiton Yan, for his patience during our many illuminating discussions. I also thank William Colocho for introducing me to my research project and his willingness to help along the way. Finally, I would like to thank Adam Edwards, Stephanie Majewski, and Mike Woods, as well as the Department of Energy Office of Science for providing the opportunity to participate in a rewarding research experience.

\section{REFERENCES}

[1] J. Irwin, C.X.Wang, Y.T Yan, K.L.F. Bane, Y. Cai, F. J. Decker, M. Minty, G.V. Stupakov, F. Zimmermann, "Model-Independent Beam Dynamics Analysis," in Physical Review Letters, Vol. 83, Issue 8, February 1999, pp. 1684-1687.

[2] Y.T.Yan, Y. Cai, F-J., Decker, S. Ecklund, J. Irwin, J. Seeman, M. Sullivan, J. Turner, U. Wienands, "Virtual Accelerator for Accelerator Optics Improvement," SLAC-PUB-11209, presented at PAC 05, May 2005.

[3] Y.T. Yan, Y. Cai, J. Irwin, and M. Sullivan, "Linear Optics Verification for PEPII using Model-Independent Analysis,” SLAC-PUB-9368, presented at 23rd Advanced ICFA Beam Dynamics Workshop on High Luminosity e+ e- Colliders, August 2002.

[4] J. Irwin and Y.T. Yan, "Beamline Model Verification Using Model Independent Analysis,” SLAC-PUB-8515, presented at EPAC 2000, July 2000.

[5] Y.T. Yan, Y. Cai, W. Colocho, F. J. Decker, J. Seeman, M. Sullivan, J. Turner, U. Wienands, M. Woodley, G. Yocky. "Precision Measurement and Improvement of e+,e- Storage Rings,” SLAC-PUB-11925, presented at EPAC 2006, June 2006. 
[6] Y.T. Yan and Y. Cai, "Precision PEP-II Optics Measurement with an SVDEnhanced Least-Square Fitting,” Nuclear Instruments and Methods in Physics Research A, vol. 558, p. 336, 2006.

\section{FIGURES}
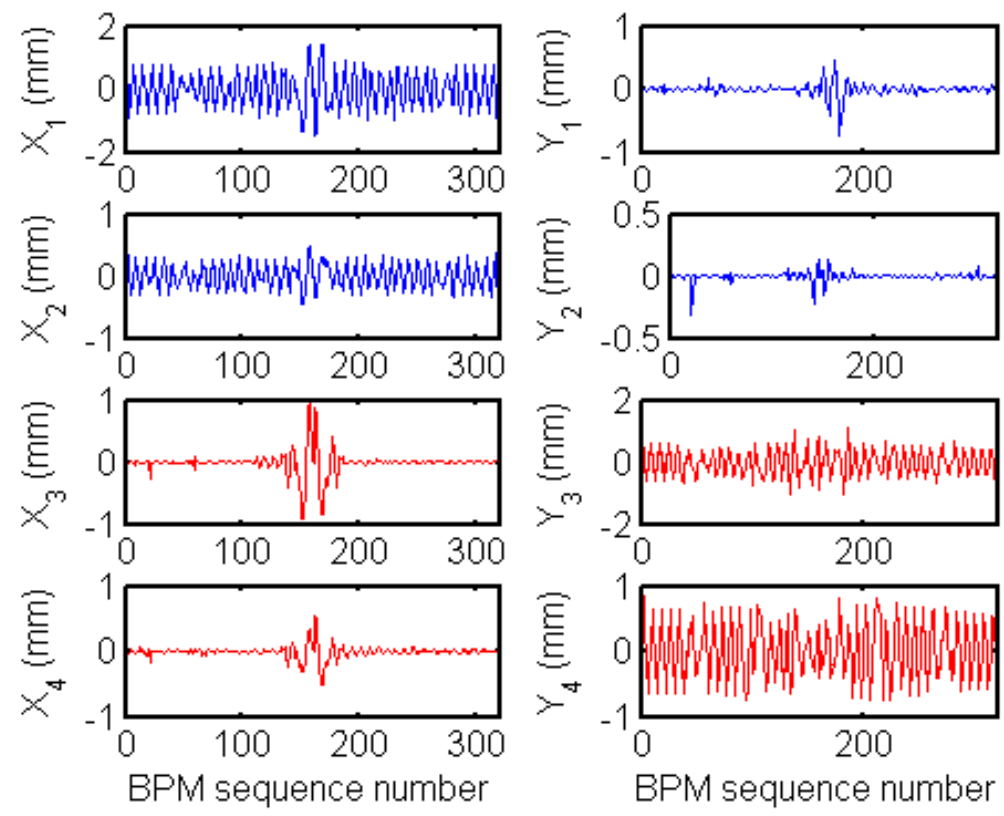

Figure 1 (color). Independent beam orbits from LER on July 27, 2006. Orbits $\mathrm{x}_{1}, \mathrm{y}_{1}, \mathrm{x}_{2}, \mathrm{y}_{2}$ are obtained during horizontal beam excitation. Orbits $\mathrm{x}_{3}, \mathrm{y}_{3}, \mathrm{x}_{4}, \mathrm{y}_{4}$ are obtained during vertical beam excitation. 

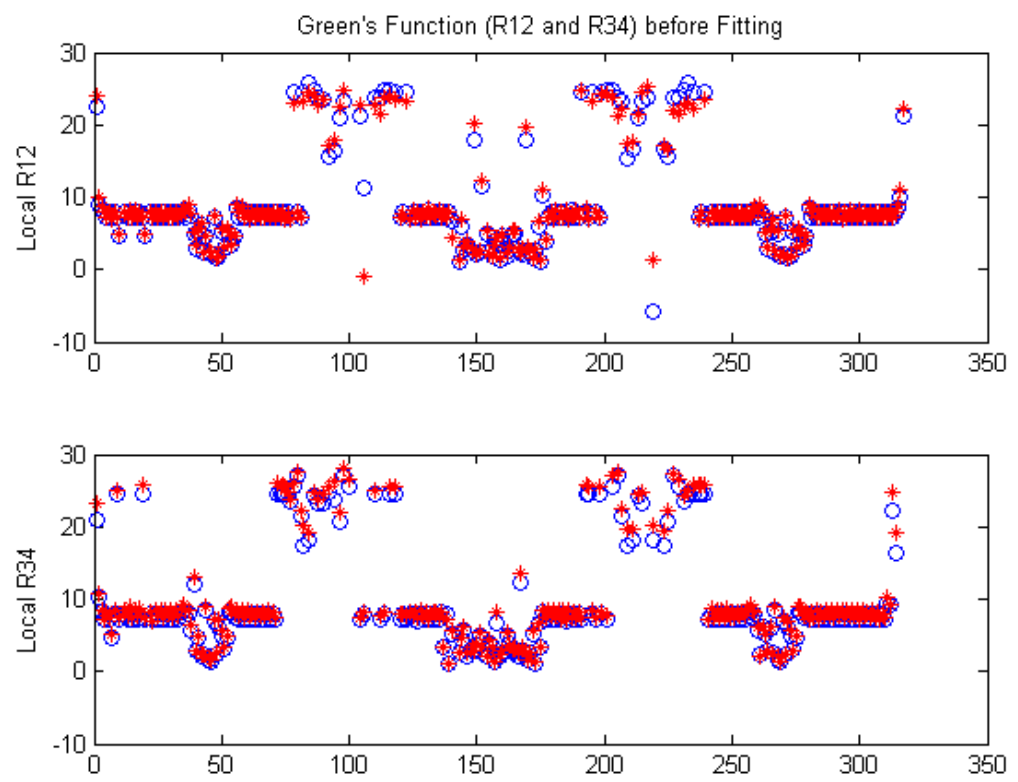

Figure 2 (color). Linear Green's functions obtained from LER. Blue circles represent functions calculated from model before any fitting. Red stars are the functions derived from measurement.


Figure 3 (color). Linear Green's functions obtained from LER. Blue circles represent functions calculated from model after SVD-enhanced fitting. Red stars are the functions derived from measurement. 

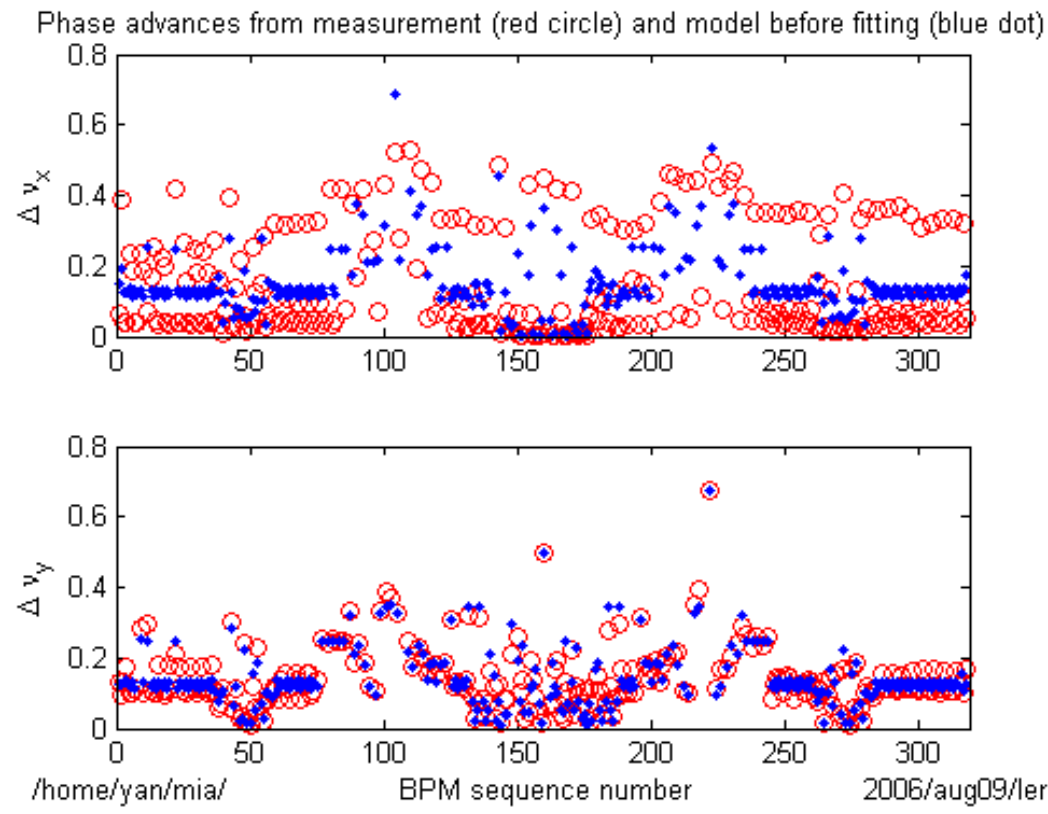

Figure 4 (color). Betatron phase advances obtained from LER before fitting. Red circles represent measured phase advances, while blue dots represent phase advances of model (ideal lattice).
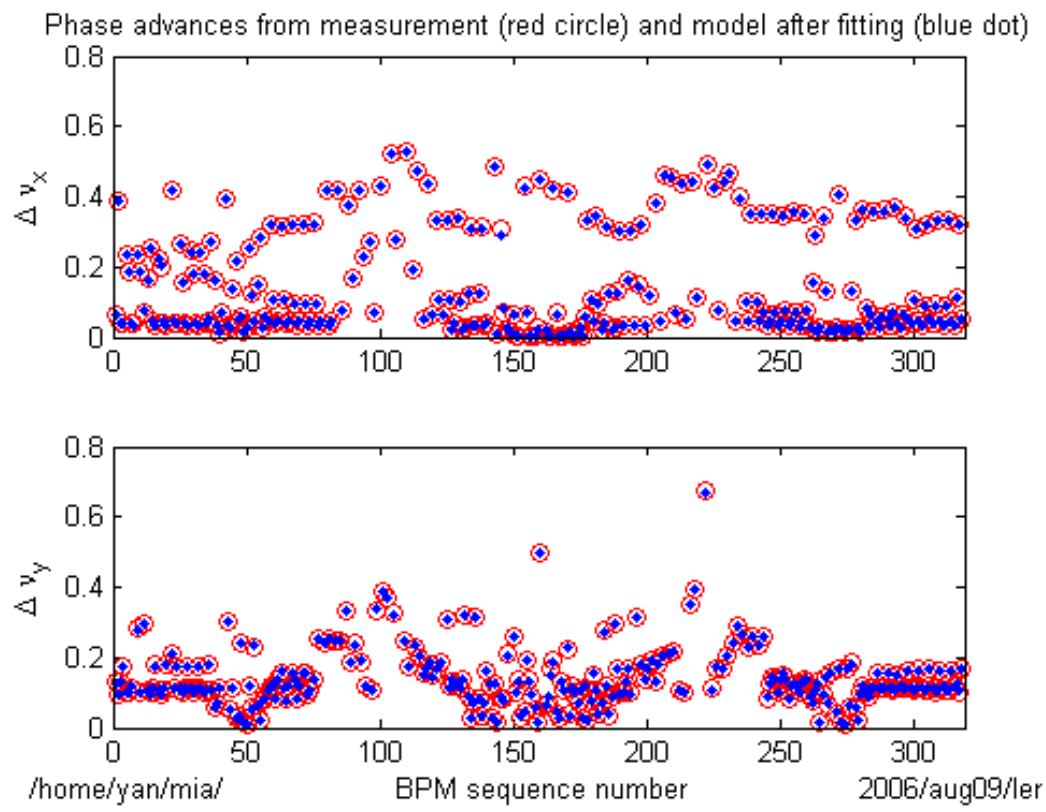

Figure 5 (color). Betatron phase advances obtained from LER after fitting. Red circles represent measured phase advances, while blue dots represent phase advances of model (ideal lattice). 

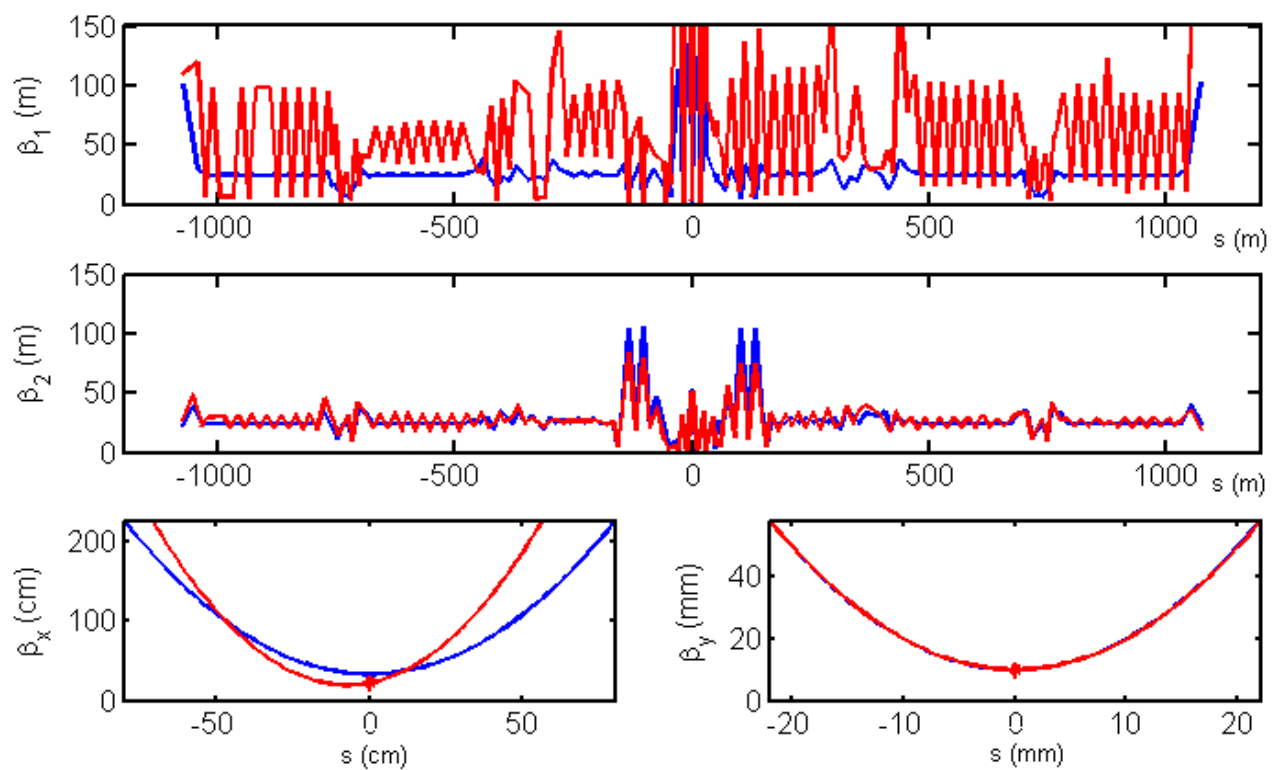

Figure 6 (color). Comparison of virtual machine beta functions (red) and ideal lattice beta functions (blue) for eigenplanes 1 and 2 in LER. Plots on bottom are beta functions at IP. $\beta_{x}^{*}=19.6 \mathrm{~cm}$ and $\beta_{y}^{*}=10.1 \mathrm{~mm}$.
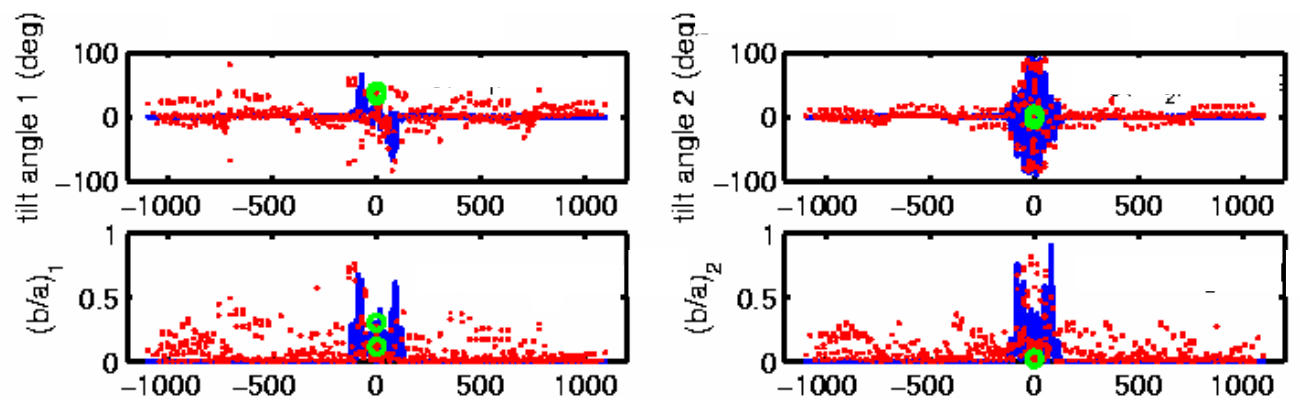

Figure 7 (color). Comparison of linear coupling parameters between model (red) and ideal lattice (blue) in LER. 

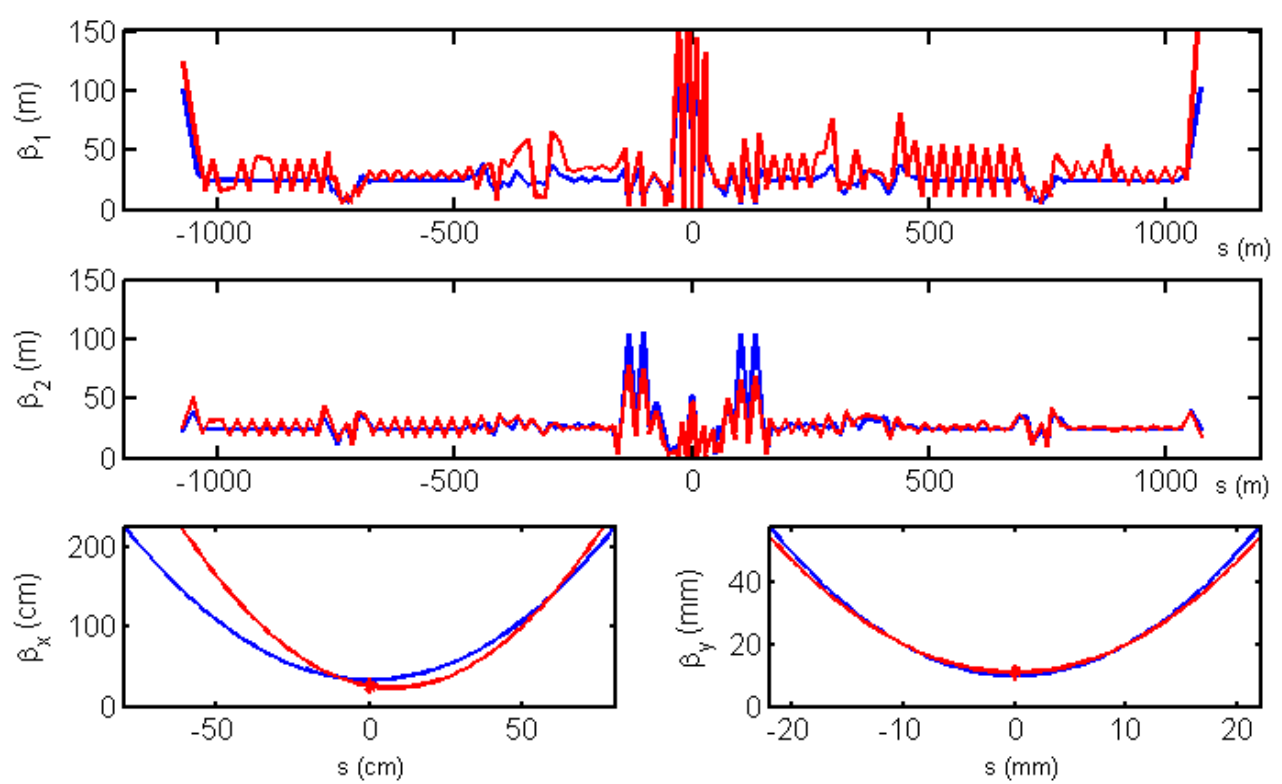

Figure 8 (color). Comparison of improved virtual machine beta functions (red) and ideal lattice beta functions (blue) for eigenplanes 1 and 2. Plots on bottom are beta functions at IP. $\beta_{x}^{*}=37.2 \mathrm{~cm}$ and $\beta_{y}^{*}=10.0 \mathrm{~mm}$.
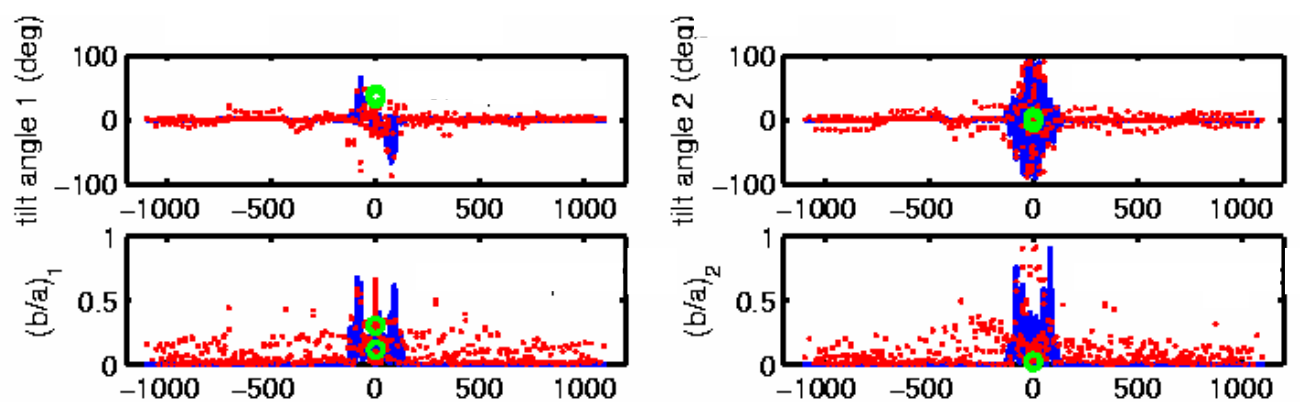

Figure 9 (color). Comparison of linear coupling parameters between improved model (red) and ideal lattice (blue). 

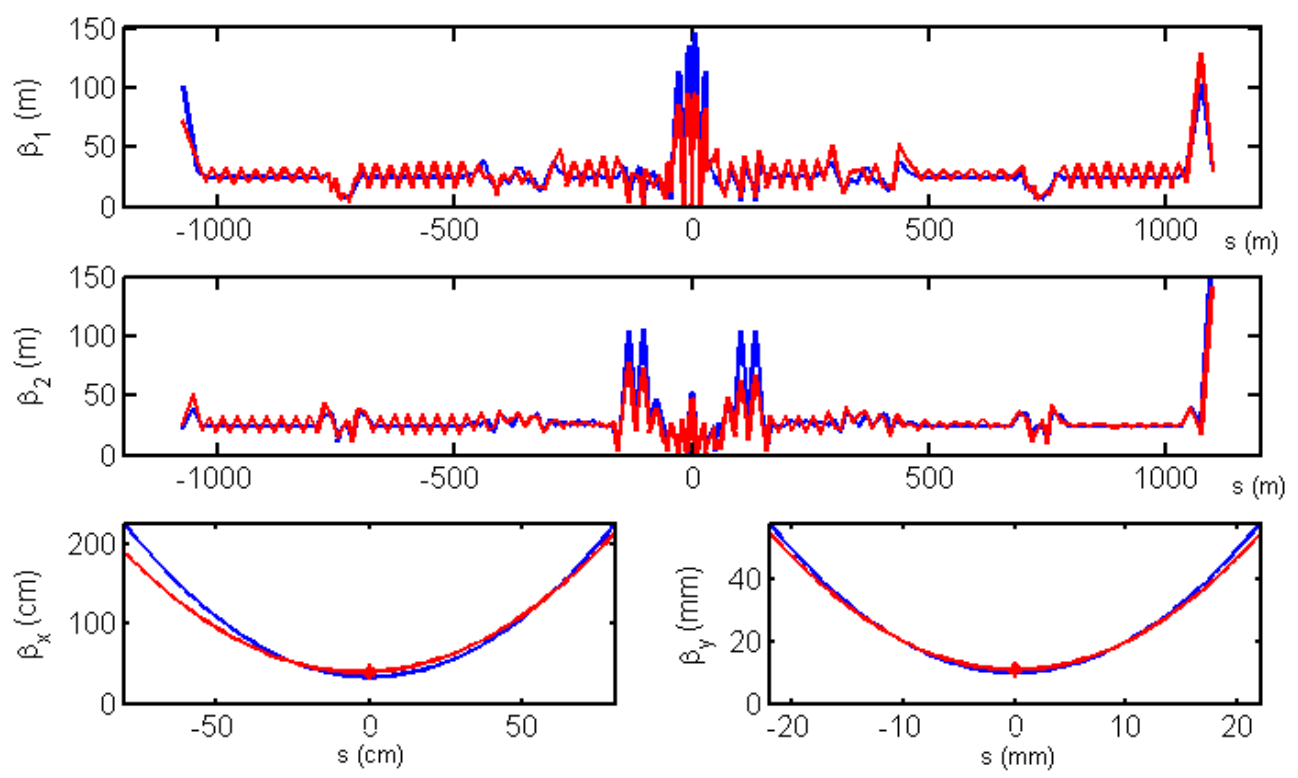

Figure 10 (color). Comparison of post-solution virtual machine beta functions (red) and ideal lattice beta functions (blue) for eigenplanes 1 and 2. Plots on bottom are beta functions at IP. $\beta_{x}^{*}=39.5 \mathrm{~cm}$ and $\beta_{y}^{*}=11.0 \mathrm{~mm}$. 